\title{
Mathematical Representations of Matte Blanco's Bi- Logic, based on Metric Space and Ultrametric or Hierarchical Topology: Towards Practical Application
}

\author{
Fionn Murtagh ${ }^{1}$ De Montfort University
}

\begin{abstract}
The Chilean psychoanalyst Ignacio Matte Blanco developed theories of subconscious processes and their relationship with conscious reasoning. This work, in the book The Unconscious and Infinite Sets: An Essay in Bi-Logic published in 1975, uses mathematical perspectives. In this article I will show how such perspectives are closely aligned with the mathematics underpinning data analytics. This is based on text analysis. Such text can be the surface expression, through expression in language, of thinking and reasoning. On the one hand, an aim of this work is to use the greatly insightful perspectives of Matte Blanco for such data analytics. Secondly a more extensive aim is to further develop the work of Matte Blanco with reference to psychoanalysis and various other disciplines.
\end{abstract}

\section{Introduction}

\section{Approaching Data and the Object of Study, Mental Processes}

In approaching the data-based study of the human mind, it is useful to note how important and how feasible it is to integrate mental processes (analytic, synthetic mental processes) with the objects of these, our human, mental processes. This is surely all the more essential when the objects of mental processes happen to be those very same mental processes.

Jean-Paul Benzécri's approach to data analysis, sometimes referred to as the French school of data analysis, displays both cohesiveness of theoretical underpinning and breadth of applications. Benzécri's early data analysis motivation, in Rennes to begin with and subsequently in Paris, came from linguistics. Benzécri's correspondence factor analysis methodology is closely associated (mathematically and in practical application) with agglomerative hierarchical clustering, and other multivariate data analysis, and more broadly, statistical analysis methods. Correspondence Analysis was initially proposed as an inductive method for analyzing linguistic data. From a philosophy standpoint, Correspondence Analysis simultaneously processes large sets of facts, and contrasts them in order to discover global order; and therefore it has more to do with synthesis (etymologically, to synthesize means to put together) and induction. On the other hand, analysis and deduction (viz., to distinguish the elements of a whole; and to consider the

${ }^{1}$ Correspondence concerning this article should be addressed to Prof. Fionn Murtagh, School of Computer Science \& Informatics, De Montfort University, Leicester, UK. E-mail: fmurtagh@acm.org

Language and Psychoanalysis, 2014, 3 (2), 40-63

http://dx.doi.org/10.7565/landp.2014.008 
properties of the possible combinations of these elements) have become the watchwords of data interpretation. It has become traditional now to speak of data analysis and correspondence analysis, and not "data synthesis" or "correspondence synthesis". In such analytics, emphasis can also be given to interactive data analysis, that includes visualization, or in one phrase, the analyst or data scientist seeks the visualization and verbalization of data (Blasius and Greenacre, 2014).

This article is structured as follows. Section 1 takes salient and important points from Matte Blanco's work. These points are of direct interest to us in what follows. Section 2 introduces metric and ultrametric mathematical concepts, how they are related to Matte Blanco's thinking, and how they permit data analytics. Section 3 considers the textual mapping, embedding in a metric space, of a short extract from a therapy session. Section 4 looks at Matte Blanco's symmetrization of our thought processes, in the unconscious, and we can relate this to conscious reasoning (invoking Matte Blanco's asymmetry in thought processes) and also to how we can find remnants or vestiges, tracks and trails, of unconscious thought processes. Section 5 turns attention to the topic of tracking of emotion. All case studies that are discussed share the underpinning mathematical data analysis platform. An Appendix discusses some further considerations in regard to textual data that can be used, in analyzing narratives (such as narratives of activities that are integrally linked to emotions).

\section{Matte Blanco's Psychoanalysis: A Selective Review}

Matte Blanco's work is concerned with conscious reasoning and subconscious thought processes. His great achievement is to develop a cognitive model that embraces both. I begin with a selective review of Matte Blanco's work in order to set out the key terms and the key thrusts of his work, such that there will be a strong resonance with the mathematical concepts and mathematical or computational processing that is discussed in this paper. Matte Blanco's The Unconscious as Infinite Sets (originally published in 1975; see Matte Blanco, 1998) was, according to the author, "written for psycho-analysts as well as for mathematical philosophers" (p. xxv) and is described in Eric Rayner's Foreword as "undoubtedly [his] most fundamental work" (p. xviii). What follows is the summarizing of particularly salient aspects of Matte Blanco's work. That will help in showing how the various points can be seen in mathematical terms. Quotations in the following are from Matte Blanco (1998).

\section{Matte Blanco asymmetry and symmetry relative to Freud's conscious and unconscious}

Relative to Freud's work, Matte Blanco had it largely reformulated in terms of symmetry and asymmetry. For him, these were "two kinds or modes of being rather than of existence" (p. 94). The interplay of symmetry and asymmetry is the focus of Matte Blanco's work. The upshot of this was that Matte Blanco arrived at what he termed a bilogical system or bi-logic. There are "two fundamental types of being which exist within the unity of every man: that of the 'structural' id (or unrepressed unconscious or system unconscious or symmetrical being) which becomes understandable with the help of the principle of symmetry; and that visible in conscious thinking, which can roughly be comprehended in Aristotelian logic" (p. 13). Freudian consciousness and unconsciousness are reformulated in terms of symmetrical and asymmetrical modes of 
being. It is to be noted that this is not a Freudian "rational-irrational" polarity but rather, on the side of the symmetric mode of being, the "unrepressed unconscious", or what is "the unconscious by its own nature or structural unconscious" (p. 95). As seen in the development of the theory of Matte Blanco, "It is an attempt at putting in logicomathematical terms the findings of Freud" (p. 49).

\section{Symmetrization gives rise to a cluster or class of things}

Within a class of things as conceptualized by the thinking person, there is perfect equivalence of class members, implying the following: no contradiction; absence of negation; displacement; space and time vanish; no relations of contiguity; arising from the last-mentioned: no order. How a class is defined in practice, or is known to the thinking person, is described in these terms. Firstly, because, as elaborated on in Matte Blanco, one class member is - in terms of class membership - indistinguishable from another class member, there is the following: "the unconscious does not know individuals but only classes or propositional functions which define the class" (p. 139). Further, "The only unity for the (symmetrical) unconscious is the class or set, in which all individuals belonging to it are included. The unconsciousness cannot, therefore, deal with parts, except by treating them as classes or sets" (p. 141). "Consciousness ... when confronted by a whole class can only consider it in two ways: either it focuses on the limits (or definition) of the class, that is, on those precise features which characterize it and distinguish it from all other classes, or it concentrates on the individuals which form the class" (p. 97).

A class comes about through condensation: "... two impulses which appear incompatible in Aristotelian logic and their union in one expression, ... is accomplished in condensation" (p. 44). The principle of generalization relates different classes. We assume various classes. Then "the principle of generalization and the principle of symmetry" (p. 11) are both taken for their explanatory capability in regard to classes. In this way, the "generalizing part [in the human] leads to symbols" (p. 106), since symbols arise out of knowledge of, or awareness of, classes. Classes are structured as, what might be called, "bags of symmetry" (in quotation marks in the original, p. 125), and also "levels". Counterposed to the symmetrical principle in Matte Blanco is the asymmetrical principle. The asymmetrical principle is visible in conscious thinking. It can roughly be comprehended in, or expressed through, Aristotelian logic: "Asymmetrical being ... perceives reality as divisible or formed by parts and, as such, related to spatiotemporality" (p. 20). Symmetrical being can by known only through the glass or prism of asymmetrical being: "Thinking requires asymmetrical relations. So does consciousness" (p. 97).

\section{Towards empiricism and analytics}

First we consider the measurement of the symmetrical. For Matte Blanco (p. 104), "Symmetrical being alone is not observable in man." Even delineating it is "already an asymmetrical ... activity". In regard to emotion, the "magnitude of emotion" is understood in terms of "the proportion between symmetrical and asymmetrical thinking" (p. 17). "[U]nconscious psychological events are not intrinsically immeasurable" (p. 18) although compared to a physical event being susceptible to just one measurement, instead with unconscious events it is a matter of being susceptible to infinite measurement - 
understood on the basis of the Cantor argument whereby a whole set, being in a bijection with a part of this same set, implies the same countable infinite cardinal for both whole and part sets. "By making the individual identical to the class, the principle of symmetry, as seen from an asymmetrical point of view, leads to the infinite set ..." (p. 106) "We must ... keep in mind the possibility that if things are viewed in terms of multidimensional space, symmetrical being can actually unfold into an infinite number of asymmetrical relations" (p. 110, footnote).

Next we consider how data may be obtained in this context. In free recall, and in other areas besides such as in literature, words are tracers for expressing what lies behind. "Consciousness cannot exist without asymmetrical relations, because the essence of consciousness is to distinguish and to differentiate and that cannot be done with symmetrical relations alone" (p. 96). "Symmetrical being is translated into asymmetrical terms by means of words. Words (i.e. their meanings) are the asymmetrical tools of the translating-unfolding function" (Italics in the original, p. 115). We have that "words, abstract things, fulfill the function of differentiating between concepts and also between other things. They are bound to be, therefore, highly asymmetrical in their structure" ( $p$. 115). To further support Matte Blanco's view here, consider the following. Text is the "sensory surface" (McKee, 1999, p. 252, formulated in statistical and computational terms in Murtagh et al., 2009) of the underlying semantics. In later sections and in the Appendix, I will return to further motivation as to why words are a good starting point for further analysis and how this can even go towards accessing aspects of underlying symmetrical being. Thus far, I have selected various central themes from Matte Blanco. This leads us to a conclusion drawn by Lauro-Grotto (2008, pp. 538-539) that directly follows from Matte Blanco: “... here comes my observation: the structural unconscious, in the way it is reformulated by Matte Blanco, the symmetric mode - all this is homologous to an ultrametric structure. The generalization principle reflects the hierarchical arrangement in which all the stimuli (or concepts) are perceived as belonging to classes, and the classes are clustered into super-classes of increasing generality. Finally, a single omni-comprehensive class is generated". Lauro-Grotto (2008) points to how equi-similar (or equi-distant) stimuli or concepts indicate an ultrametric (or hierarchy, or tree) topology.

\section{The Metric Space and Ultrametric, or Hierarchical, Space Structures that Underly Matte Blanco's Asymmetry and Symmetry}

\section{The real world, metric space: The context for asymmetric mental processes}

Our real ambient space, and an ordered timeline, are fundamental to our human, conscious mental processes. A metric perspective is very appropriate for our usual threedimensional world around us, to which we can add time as a fourth dimension. The real number line is then appropriate for any dimension of this 3D+T (three-dimensional space, and time) world. For geometric modelling, Cartesian coordinates can be availed of where this appellation comes from mathematician and philosopher, René Descartes (15961650). 
For our purposes, the terms metric and distance are used synonymously. The visually intuitive distance, informally expressed as the "as the crow flies" distance, is the Euclidean distance. In examples in sections below I will be using the Correspondence Analysis methodology. This data analysis technique is, in fact, a highly developed platform or environment for data analysis. It begins with appropriately coding the data that expresses what we are interested in. The data starts as symbolic and/or numeric, and is taken into a categorical (also termed qualitative) data matrix for analysis. Metric geometric is appropriate for semantic analysis. For example, in textual analysis, text segments are defined as the average of their constituent words, and words are defined as the average of their constituent text segments. We embed the text and words in a metricEuclidean - latent semantic space. A high point of such semantic analysis, using metric embedding, can be seen in Bourdieu's use of Correspondence Analysis. Renowned social scientist, Pierre Bourdieu, 1930-2002, used Multiple Correspondence Analysis for mapping survey data into a metric, latent semantic space. This was done in various works including his book, Distinction, A Social Critique of the Judgement of Taste, published in French in 1979, and in English in 1984. Bourdieu's work relates to social and aesthetic preferences, cultural and educational capital.

In practical and operational settings we can take text, or text transcribed from voice, or survey or questionnaire results, or other measured data on our observables, together with the characteristics of our subjects, or observables, and map such data into a semantic space that is metric (because, by design, the factor space, or latent semantic space, is a high dimensional metric space). Then we can proceed from there to induce a hierarchy, or tree structure. Distance on a tree structure, or hierarchy, is more restrictive than, for example, Euclidean distance. Distance on a tree structure or hierarchy is termed an ultrametric, and we will look further at it in the subsection to follow. For a metric context, the natural geometric ordering is on the real line, whereas in the ultrametric case the natural ordering is a hierarchical tree. Khrennikov (2014) notes how the real number system is used for measurement in real world, physical spaces. But ultrametric distances are appropriate, Khrennikov holds, for mental spaces. See Khrennikov (1997, 2004, 2007, 2010) for a very great amount of work studying dynamical processes in ultrametric spaces. Thus, Khrennikov lays the basis for unconscious information processing. Khrennikov (2014) notes the following: "The idea that physical and mental spaces have essentially different geometries was discussed already by Aristotle. He emphasized continuity, infinite divisibility, and connectivity of the physical space. At the same time he presented motivations that the mental space is discrete, hierarchic, and totally disconnected. The latter matches perfectly with the modern notion of a totally disconnected topological space."

\section{Ultrametric topology, relevance for expressing symmetric mental processes}

Having surveyed Matte Blanco's view of unconscious thought processes expressed as (Matte Blanco's term) symmetry, and conscious reasoning expressed as (again Matte Blanco's term) asymmetry, in this section I will lay out a basis for mathematically modelling these - symmetry, asymmetry - as respectively ultrametric (i.e., metric on a tree or hierarchy) and metric. 
As observed by Lauro-Grotto (2008, p. 539), the aspect of anomaly modelling via an ultrametric is nicely consistent with Matte Blanco's symmetrical logic: “... we know that something similar can actually be experienced in finite space when we look at a very distant three-dimensional structure and we perceive it as though it were a single point. Symmetrization of relationships can therefore be described as a transition from a metric to an ultrametric conceptual organization". Take the example of Figure 1. We consider objects $y$ and $z$ to be agglomerated, i.e. clustered, at level 1.0, and then a third object, $x$, to be with them in a cluster containing all at level 3.5. This is a standard dendrogram representation. The root node is at the top and the branches are at the bottom. From the hierarchy or tree, the distances between $x$ and both $y$ and $z$ are equal to 3.5. This is their ultrametric distance. One among an infinite number of depictions of this set of relationships is in the right panel of the figure.

In an ultrametric space all triangles are either isosceles with small base, or equilateral. We have here very clear symmetries of shape in an ultrametric topology. In Figure 1 from $x$ 's point of view, $y$ and $z$ are indistinguishable. In mathematical terms, an ultrametric topology (which we can quite acceptably term a hierarchical topology, or a tree topology) has further properties that are recognizably along the lines of Matte Blanco's symmetric mental processes. Insofar as a cluster, corresponding to any node of the hierarchical tree, contains a set of objects, these objects are all identically members of this cluster. There are unusual properties. Any member of the cluster can be taken as its centre. As a mathematical ball or hypersphere, the radius equals its diameter. The cluster or ball is topologically open and closed as the same time and this is termed a clopen set. This last mentioned property is explained thus: the set is closed because objects on its boundary can be members; and it is open because the cluster extremity is defined by what is not a member relative to the external, complement set. These are deep mathematical concepts, from topology and then other areas such as number theory. Our essential point here is that the mathematical viewpoint is so greatly helpful towards taking into practice - the practice of data analytics to start with - the exceedingly insightful work of Matte Blanco. 

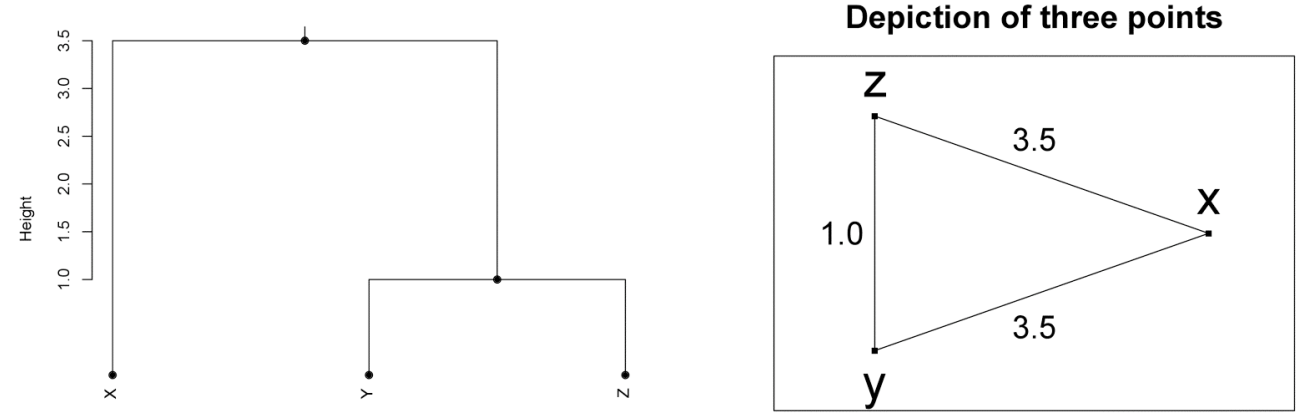

\section{Figure 1}

Left: Hierarchical clustering of $x, y, z$. The strong triangular inequality defines an ultrametric: every triplet of points satisfies the relationship: $d(x, z) \leq \max \{d(x, y), d(y, z)\}$ for distance $d$. Cf. by reading off the hierarchy, how this is verified for all $x, y, z$. In addition the symmetry and positive definiteness conditions hold for any pair of points. Right: A depiction of these three points. They form an isosceles triangle with small base. The base is formed by points $y, z$.

In the practical and operational context of analyzing data, inducing an ultrametric means building a hierarchical clustering from given data. A mapping of metric to ultrametric is achieved by an agglomerative hierarchical clustering algorithm, a well-established approach that depends on a cluster (compactness, or connectedness, or other) criterion.

Finally, in this short discussion of hierarchical clustering, to draw yet another link to the work of Matte Blanco, it is noted in Rayner's $(1995$, p. 2) review of Matte Blanco that the latter's investigation of "process of thinking ... emphasizes the essential centrality of classificatory activity at all levels of thought, even in the unconscious".

\section{Mapping A Short Discourse from a Therapy Session}

Matte Blanco considers the infinity of human thought processes. Now from some form of expression of human thought, I first address how our data analytics allow us to establish a spatial mapping of that. A metric space is our initial aim in regard to this mapping. The dimensionality of the metric space can be arbitrarily high. By metric, it is meant that all entities in this space are endowed with a distance, a typical one being the Euclidean metric. The Euclidean distance, the "as the crow flies" distance, is visually intuitive for us.

Notationally, we have the following. For objects that we are dealing with, $x_{i}$ and $x_{i}$, consider them as positioned in a space of dimensionality $J$, that is endowed with the

Euclidean distance. Their (Euclidean) distance squared is: $d\left(i, i^{\prime}\right)^{2}=\sum_{j=1}^{J}\left(x_{i j}-x_{i i^{\prime}}\right)^{2}$

In our discussion here I will explain the overall aims and implementations of our 
analytics. In analyzing textual narratives, Murtagh et al. (2009) explain a great deal more about the underpinning mathematics of our methodology. Murtagh (2005), in particular chapter 5, covers many themes ranging from classical Greek philosophical texts, Russian literature, contemporary political discourse and many other areas including an extensive study of dream reports that will also be discussed in the section to follow below. The expression of human thought processes that will be used here is textual. That can easily enough be the outcome of a recorded expression of vocal, gesture or other human activity. Then taking the words used in their context, the aspect of context that we particularly use is the presence/absence and frequency of occurrence of words in their surroundings. In this way, the semantics or meaning of a word is the totality of the contexts in which it is used.

\section{Language aspects of discourse in therapy}

Schneider (2013) presents an extensive review of structural frameworks for a therapistpatient discourse. He starts with consideration of the analyst's narrative vis-à-vis the patient's narrative. In therapist-patient discourse, "linguistic strategies", comprising substitutions of words and phrases, or other forms of reformulation, are used by the therapist in the discourse in order to influence and modify the patient's narrative. This works on different linguistic levels. Now, every such therapy session is embedded in a social context. Therefore the socio-cultural practices imply that meaning and understanding are influenced, and indeed created, by sources that are external to the therapist-patient session. Thus we ask the following, with a view towards later, comparative assessment of therapy sessions. Can we map out a therapist-patient discourse, in a way that is revealing and illustrative of the discourse flow? Such a mapping should be illustrative, in a visualization or display sense. Further afield, then, with lots of therapy sessions undergoing the same sort of analysis, aims are as follows. Firstly, an aim is to extend beyond the illustrative, in order to be suggestive of, and hence reinforcing, good practice. Secondly an aim is to determine heuristics for successfully addressing problems.

An exchange in a therapy session is recounted by Schneider. The patient is considering termination. Schneider describes in detail the many connotations and denotations of what is said in the short discourse, consisting of 34 exchanges ( 17 each, in turn) of patient and therapist. In this very detailed commenting on the therapist-patient discourse, Schneider notes the importance of "verbal quirks in tone and grammatical form, departures from normal discourse rules, truncated utterances, etc.". The mutual conditioning of connotations of words is noted, and external socio-cultural "rules". Elements of silence are important. Tone is important, and Schneider discusses the different meanings that apply to the term "OK". Speed of vocal exchange in discourse means that the interaction is ad lib, but against a given background. The foregoing points to our interest in being able to map out the discourse, in a way that lends itself to visualization of such discourse. Such mappings should be reflective of the data, and not impose any structural model. Schneider refers, a few times in his detailed discussion, to how his own verbal expressions were counter-transferential. He also notes how the discourse is to be viewed here in terms of measures of awareness in the exchanges; the significance for one another; how each values the other; and the importance of one for the other (how important the therapist is to the patient, and how central and hence important the patient is to the therapist). So there is the possibility to consider value, hence measurement and 
even, if really pushed, quantification. We will consider this as part and parcel of our mapping of the discourse. Preserving "face" and self-esteem are just among the examples of how value enters into the discourse. So too are the "force(s) of utterances" and the kind of speech act that the utterance is. For Schneider, "linguistic aspects of the exchange" move into emotion. And: "In the whole emotional context of the relationship the words function not merely as bearers of quasi-propositional meaning but as emotional gestures, as actions." (Schneider notes that what is "extra-intentional" is not to be taken as coterminous with the "unconscious'." This is very clear from the earlier discussion of socio-cultural context, just as one example of the conscious, albeit very complex, domain of what we are considering.)

\section{From the discourse text to the mapping}

Schneider (2013) provides 17 patient and therapist exchanges. The first two are as follows:

1. "1P", "Sigh Well dots I know I have to leave (falling tone) dots (Long pause in which the patient looks away and then at me.)"

2. "1T", "Ah, hmm dots How do you know that?"

3. "2P", "(Looks slightly taken aback) I mean dots I guess it's time for me to move on."

4. "2T", "What makes you guess? Is that something you can guess?"

In the previous subsection, we have noted many levels of meaning of words and other discourse elements (like pauses, here noted as such, or alternatively noted with a succession of dots). Since we will not consider punctuation (taking it to be of far less interest than the words - but note one exception that we allow, for question marks), and with some word pairs being useful to keep as a pair (e.g. a verb accompanied by "not"), and to cater for non-standard word usage (included here were "Whyzat?", "Yeahh", "yeah", "Weeeeeell") we elected to do the following. We just retained a selection of words, and in a few cases converted the given word to a substitute word. We did such substitution in analogy with the fairly standard practice in text analysis to carry out stemming and lemmatization (e.g., "go" is used in place of "going", "goes", etc.). So we retained, or converted, words as follows. Because of the value-related discussion in the previous subsection, we kept verb stems, although we ignored most uses of the verb "to be". Verbs of motion, for example, lend themselves to labelling with some sort of value (e.g., "fall" or "leave" being negative). Because of positive or negative meaning, we kept "yes" (and substituted this for "yeah", "yeh", etc.), "no", "OK", "right", "sure", "certainly", "nod" (substituting "nods"), "tired", "afraid", "fixed", "smile" (substituting "smiles"), "laugh" (substituting "laughs", "half-laugh"). We kept because we also wanted to see if there would be any difficulty in regard to our analysis with them, "goodbye", "oh", "ah", personal names - here, "Z", "Ed", - "silence", "mother", "pause" including dots. Finally, given the great importance of the subjective and related factors here, we retained: "I", "me", "myself", "you", "your". Finally, due to the importance of interrogative mode here, we kept the question mark, as a "Q".

The first four utterances thus become the following (cf. above):

1. "1P", "sigh pause I know I haveto leave falling pause looks me"

Language and Psychoanalysis, 2014, 3 (2), 40-63

http://dx.doi.org/10.7565/landp.2014.008 
2. "1T", "ah pause you know Q"

3. "2P", "looks I mean pause I guess me move"

4. "2T", "makes you guess Q you can guess Q"

Our input data consists of the selected set of words (just defined as a contiguous sequence of one or more characters) in each of the 34 utterances. The utterances are also labelled with the "P" or " $\mathrm{T}$ " for, respectively, patient or therapist. Mathematically we consider two dual (meaning integrally related) spaces, the set of 34 utterances in, as we find, a 91dimensional space where each coordinate is a word; and the set of 91 words, in the utterance space, such that each word has a coordinate value on the 34 utterances/coordinates. We speak of the cloud of 34 utterance points in a 91-dimensional space, and the cloud of 91 word points in a 34-dimensional space. In the standard mathematical way of defining how one of these spaces is related to the other, we have a matrix that crosses the utterance set (providing the rows of the matrix) by the word set (providing the columns of the matrix). That is what a matrix is, a transformation between vectors in one space and the set of transformed vectors in another space. The intersection values of the matrix are the number of times that a given word appears in a given utterance. Not surprisingly most of the intersection values are zero, the given word is not in the given utterance: occupancy is $6.9 \%$.

We endow the space of utterances, and the space of words, with the chi squared distance. Then we transform the two clouds in the dual spaces endowed with this chi squared distance into a factor space, where the two clouds of points are projected into the same factor space, that is endowed with the Euclidean metric. That certainly facilitates visualization of the semantic relationships, arising out of relative positioning in the factor space. The coordinate axes of this space, i.e. the factors, are ordered in decreasing order of importance. "Importance" is the contribution to inertia. The contribution by inertia of a cloud of points about the axis to the inertia of the whole cloud betokens the classical mechanics, developed by Christiaan Huyghens (1629-1696). One aspect needs to be noted: we have coordinate, hence positional, information for our points, utterances and words. We have their masses also, provided by relative frequencies of marginals of the utterances times words matrix. Mathematically then the relative importance, the contributions to inertia, of the factors are given by the decreasing eigenvalues of a matrix product based on our input data, the utterances by words matrix.

To show the latent semantics expressed by the factors, it is generally the case that the plane of factors 1,2 , followed by 3,4 , etc. are perused. We find that factor 1 is strongly related to the 9th utterance, and factor 2 to the 8 th utterance. The information content of these factors is respectively $7 \%$ and $6.7 \%$ of the total cloud inertia (the same, from the mathematical principles involved, for the cloud of utterances and the cloud of words). We prefer the layout, and expressiveness, of the plane of factors 3,4. As can be seen, in Figure 2, the information content of these factors, $6.15 \%$ and $6.07 \%$, is not much lower than that of factors 1 and 2. We have prepared Figure 2 to avoid overcrowding, with labels of 91 words, and numbers associated with the 34 utterances. So in Figure 2 we took just the 14 highest valued utterances, and the 14 highest valued words, relative to their coordinates (i.e. projections) on the two factors here. All words have their locations at the small red triangles. The unlabelled words, as seen, are bunched at the origin (i.e. the coordinates 0,0 in the plane of factors 3,4 ). The 14 labelled words are to seen in red. 
The utterances all have a blue dot. The bunched utterances at the origin have the words superimposed (so the blue dots there are superimposed by the red triangles). We have cut down on the utterances and words shown in order to direct our attention on the most useful and revealing subset.

We see how factor 3, the horizontal axis in Figure 2 is characterized essentially between the extremes of utterance 15 , and utterance 23 . Utterance 15 , indicating that there is no sense in continuing her therapy, is a low point in the dialogue. (See pp. 13-14 of Schneider, 2013, where he discusses this utterance by his patient.) Utterance 23 is the patient categorically backing up that position. Now let us move to the vertical axis (called the ordinate, while the horizontal axis is called the abscissa). To the positive side we have the distancing of the patient from the therapist, exemplified by her utterances 15 and 23; and then the somewhat cooling of that distancing towards the end of the dialogue. (The ending of this dialogue was to see each other once again in a therapy session one week later. Schneider, 2013, reports however that the patient ceased the therapy two months later.) The two utterances, 15 and 23 , are initially as follows:

-Utterance 15: "8P", "Well yeah sort of. It trivializes the whole thing."

-Utterance 23: "12P", "I'm thinking of how I'm tired of going over the same stuff."

One important issue relates to the origin. This is the average, more correctly the centre of gravity, of the utterances cloud of points, and of the words cloud of points. As always here, we are dealing with a projection onto a low-dimensional space, a plane. As the average, what is projected to, or very close to, the origin is not particularly interesting to us. 


\section{CA factor map}

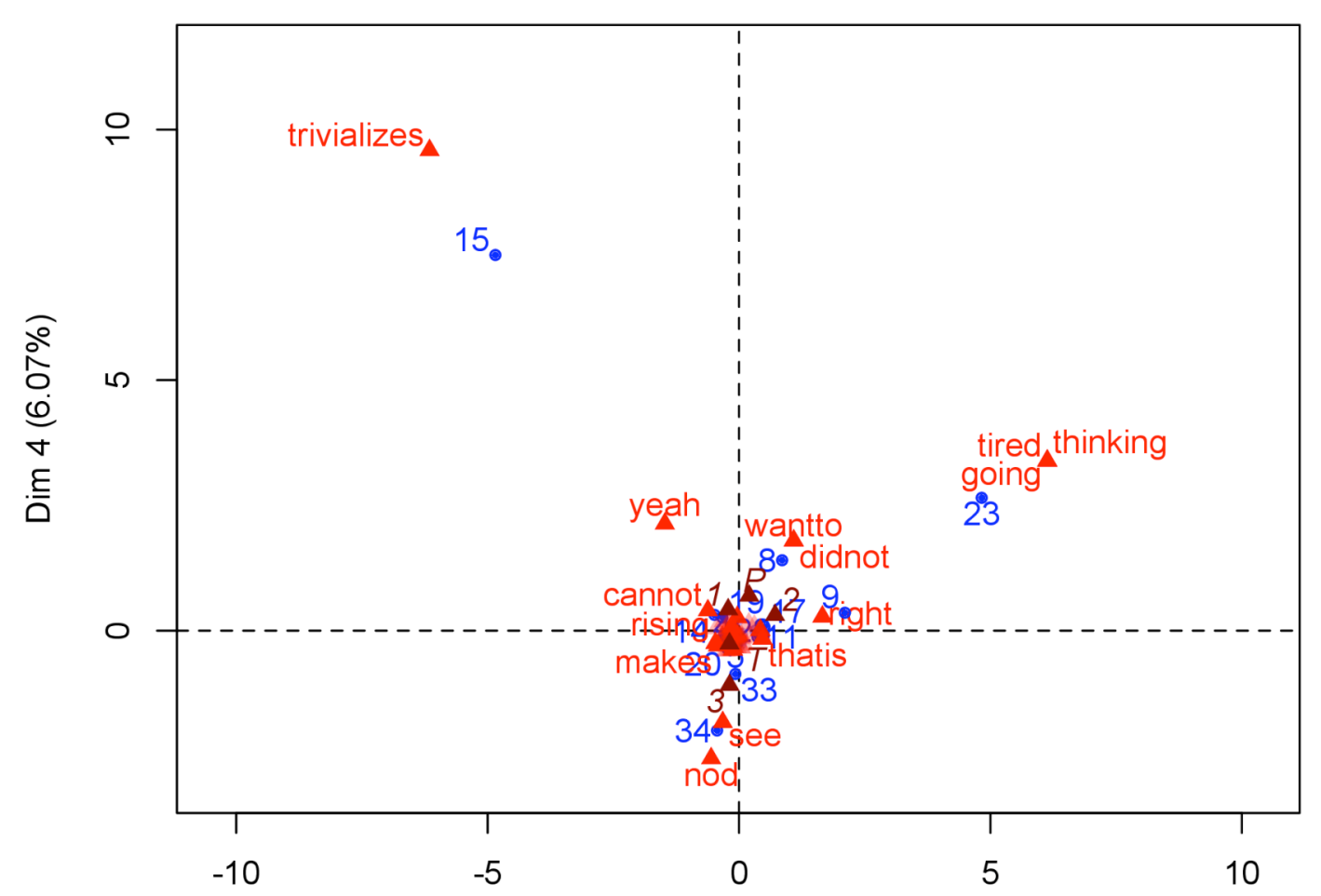

$\operatorname{Dim} 3(6.15 \%)$

\section{Figure 2}

A display, showing the factor plane of factors 3 and 4, of Schneider's (2013) therapy session fragment. The red triangles here are the 91 words that were retained. The blue dots here are the 34 alternating utterances of patient and therapist. Labels are the 14 highest coordinate, on either axis, utterances, and the 14 highest coordinate words. Shown as supplementary element projections are the patient and therapist, $\mathrm{P}$ and $\mathrm{T}$ respectively; and the three segments determined as being statistically significant for the narrative flow here. These are close to the origin, labelled respectively 1, 2, 3 (coloured brown, as are the labels $\mathrm{P}, \mathrm{T}$ ).

In summary the mapping here is one of, firstly, assertion of the patient's consideration of ending the therapy, contrasted with her conciliatory justification of this; and secondly, the patient proposing to leave the therapy versus a relatively accepting state at the end, to return to the next therapy session. To aid interpretation, we plot so-called supplementary elements, meaning elements projected into the factor space post-analysis. These supplementary elements are projected at locations shown by the brown triangles. In Figure 2, just a little away from the origin, we see the patient $(\mathrm{P})$ located to the positive side of factor 4 , thereby understandably with this half axis being characterized by her considering to end the therapy. We have the therapist a little to the negative side of this factor, factor 4, being more concerned (for lots of reasons, discussed in Schneider, 2013) with continuing therapy. 
One aim of applying clustering to the narrative flow in a context such as this therapy session is to segment the flow. As exemplified in Bécue-Bertaut et al. (2014), this can be done on a statistical basis by permutation testing of what is allowed to be clustered, subject too to the clusters respecting the chronological order of the utterances. The clustering, in order to be consistent with the inertia-based factor space construction principles should use an inertia or variance (i.e. the inertia when all masses are identical, as they become when projected into the factor space) principle. We must relax this requirement to the well-known complete link agglomerative criterion, in order to ensure that we can construct a hierarchical agglomerative clustering (for full discussion, see Murtagh et al., 2009, or Bécue-Bertaut et al., 2014; what is at issue is that the agglomerations creating clusters are representable by a hierarchy, with no inversions, also termed reversals, in levels of the hierarchical tree). The complete link agglomerative criterion for cluster creation is also a cluster compactness criterion, that is often in practice close to the inertia or variance (i.e., inertia with unit masses) agglomerative criterion. Here, as stated, we build the hierarchy just as far as we are allowed by our statistical testing for the hypothesis, at each agglomeration, as to whether there is significant "sameness" in the cluster. With reference to the planar projection of our high dimensional space (the one factor space resulting from the utterance space and the word space), it is to be noted that our clustering is on the basis of the full factor space dimensionality.

We find the following outcomes in regard to segmenting the flow of, in total, 34 utterances: from utterance 1 to 22 , from 23 to 30 and from 31 to 34 . Utterances 22 and 23 , and then 30 followed by 31 to 34 , are as follows. We see the change in flow between utterances 30 and 31; and the somewhat more relaxed ending in utterances 31-34. See now in Figure 2 how segments 1 and 2, while as for all segments are fairly close to the origin, nonetheless point to, respectively, the patient's making of her case (upper left, i.e. positive F4, negative F3), justifying it (upper right, i.e. positive F3, positive F4), and then the third segment associated with the somewhat conciliatory ending (negative F4).

•22: "11T", "What's up?"

-23: "12P", "I'm thinking of how I'm tired of going over the same stuff."

-30: "15T", "(after another long pause) Well, look here, if you're asking me whether you're fixed, whether you graduate now with your BMH, I'll tell you what I've told you before. You've made a lot of progress, it's naive to think that there'll come some definitive point at which you're fixed, no more problems. I'm sure you could continue this work on your own; I think you'll be OK without therapy, but it's up to you. It's what you want to do dots (pause) But, now, if you're asking me will I miss you? The answer is yes. But I'm certainly not going to tell you what you should do, (tone of mock desperation), cause there ain't no should."

-31: "16P", “OK. (long pause)"

-32: "16T", “OK (long pause)"

•33: "17P”, "I'll see you next week."

•34: "17T", “(nods) See you then."

Another interesting thing we can do is look at the statistically most significant words associated with categories, like $\mathrm{P}$ (patient) and $\mathrm{T}$ (therapist), or the segments in the 
narrative flow. Significant over- and under-representation of words in the category are determined. Interestingly, for the patient, two words in particular are very significant, over-represented being "I", and under-represented being "you". For the therapist, it is just the other way around: "you" is over-represented in the therapist's utterances, and "I" is under-represented. Coming now to the three segments of the therapy session narrative flow, for the first segment (utterances 1-22) we find just the word "you" being underrepresented. For the second segment (utterance 22-30) we do not find any word being statistically significant. For the third segment, we find the words "see" and "ok" being significantly over-represented.

This, therefore, completes, for now, our analysis of the short therapy session dialogue. While there is great depth and valuable outcomes from Schneider's discussion, nonetheless for large-scale assessment there is a need to focus our attention, to have visualizations, to find statistically significant relationships, and, all in all, to prioritize our analysis steps and analysis outcomes.

\section{An Approach to Detecting Matte Blanco's Symmetry in Bi-Logic}

The factor space is a latent semantic space. It provides an appropriate platform for further analysis work on the data. While our focus is on textual data, it is clear that activities and actions can be considered also. In the therapy session, we used indications of pauses, for example, and of laughing. At least in terms of no restraints, and mathematically, our factor space can be of arbitrarily high dimension. Our data mining analysis methodology is (statistically) robust, and (computationally) scalable. One of the studies carried out in Murtagh (2005, see section 5.10.5) relates to 910 texts comprising 931,537 words. For all data analysis processing work in this area, we very much recommend that the first stage of the processing is the creation of the factor, latent semantic, space.

\section{Dream reports: Seeking vestiges of the subconscious in data}

From the Dreambank repository (DreamBank, 2004, and see also Domhoff, 2002, 2003, 2006) I selected various collections. See Murtagh $(2012 a, b)$ for further description and analyses. One set of 139 dream reports, from one individual, Barbara Sanders, was particularly reliable (according to Domhoff, 2006). In order to have a text that ought to contain vestiges of ultrametricity because of subconscious thinking, admittedly subconscious thinking that was afterwards reported on in a fully conscious way, I took these Barbara Sanders dream reports. In discussion of this data provided in Domhoff (2002) he notes that there is "astonishing consistency" shown in dreams such as these over long periods of time. Taking the set of 139 of the Barbara Sanders dream reports, I used the 2000 most frequently occurring words used in these dream reports including function words. Then I took 30 words to carry out some experimentation with their ultrametric properties. These are listed as follows. Note that the processing converts all upper case to lower case. The thirty words selected were as follows: "tyler", "jared",

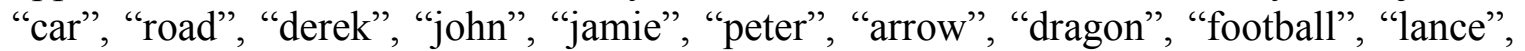
"room", "bedroom", "family", "game", "mabel", "crew", "director", "assistant", "balloon", "ship", "balloons", "pudgy", "valerie", "dolly", “cat”, "gun”, "howard", "horse". These words were selected in order to have some personal names, some words that could be metaphors for the commonplace or the fearful, and some words that could be commonplace and hence banal. Names of people are: Tyler, Jared, Derek, John, Jamie, 
Peter, Lance, Mabel, Valerie, and Howard. I carried out, firstly, a Correspondence Analysis of all 139 dream reports crossed by the 2000-word set. Then I use for the subsequent analysis the Euclidean, factor space, with full dimensionality, for the 30 selected terms. Figure 3 shows a hierarchical clustering of these terms, using their fulldimensionality factor space coordinates. That is to say, the 30 selected terms are used in their semantic embedding, or in accordance with their latent semantic expression.

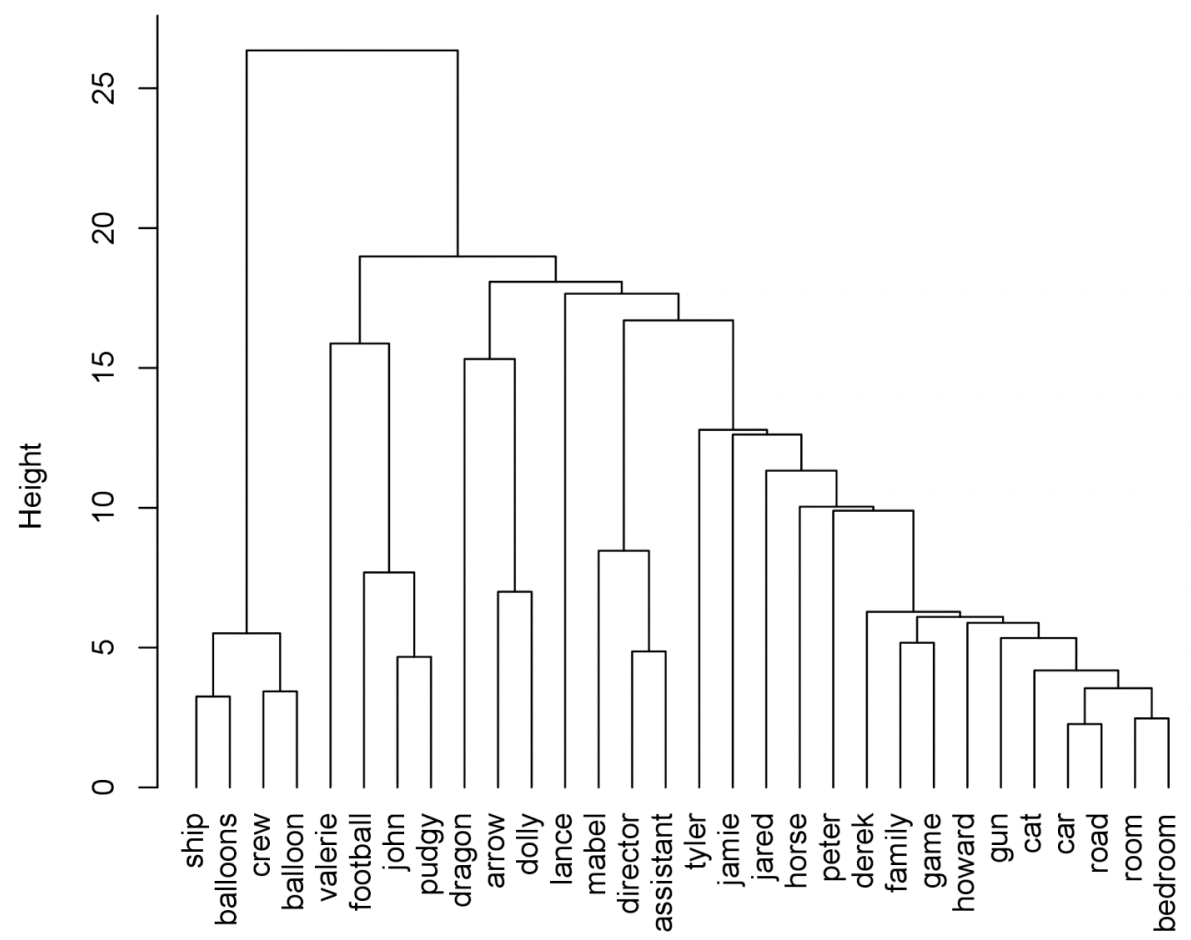

Ward

\section{Figure 3}

Hierarchical clustering using the Ward minimum variance agglomerative criterion. Note how "car" and "road" are clustered early on in the sequence of agglomerations. Note similarly "room", "bedroom". In the first four terms that are clustered here, we note the interestingly mixed, but nevertheless mutually close, semantics of "ship", "baloons", and "crew", "baloon".

Forming a hierarchical clustering in this way, in the traditional manner, can be helpful in understanding all important similarities in one's data. The clusters themselves can be explored (i) with respect to cluster membership, and (ii) with respect to the role as a cluster centre of gravity location in the factor space. It has also been traditional practice with Correspondence Analysis to explain the meaning, and interpretational relevance, of the factors in terms of the clusters, and vice versa, to explain the clusters in terms of the 
factors. (See Murtagh, 2005.) However we can, and should, note the following issue. A hierarchical clustering, such as is shown in Figure 3, is induced on the data. From any data set, of coordinates or of a dissimilarity or similarity measure, we can construct a hierarchical classification. In order to find strong associations that could lead to illustrating the subconscious of Barbara Sanders, we can draw on Matte Blanco in the following way. We will seek inherent ultrametric relationships in the data, starting from, and only that, induced hierarchies. Our procedure is as follows (Murtagh, 2014b). We use two or more hierarchical clusterings, and form their consensus, in order to bring us closer to what is inherent to the data and not just imposed. Our procedure for constructing a consensus hierarchical clustering is innovative. We examine relations in triplets of points (here, the 30 selected words), which, because they come from a constructed hierarchical clustering, respect the ultrametric inequality.

We further restricted our word set to 16 words, to especially focus on named individuals. These were as follows: "Peter", "Mabel", "horse", "arrow", "John", "pudgy", "Darryl", "football", "Derek", "game", "Howard", "gun", "family", "Dwight", "car", "road". Explanation for some of these follows. The dreamer, Barbara Sanders, had an affair in the past with Derek; Howard was her divorced, now deceased, ex-husband; Darryl was an exboyfriend; Dwight was her favourite brother; John, a young man, she fantasizes about; Peter was a student, and Mabel was a co-worker; horse, arrow, and especially gun were used metaphorically quite often. For all of these 16 words, there was quite sufficient reported presence in the dream reports used.

We determined the consensus hierarchical clustering (using the full dimensionality factor space, and in order to have quite different agglomerative clustering criteria, using the single and complete link criteria). Murtagh (2014a) presents a summary of this work, and Murtagh (2014b) provides full details. Based on the consensus hierarchy, we sought corroboration, in the factor space coordinate data, hence in the Euclidean latent semantic space, for ultrametric-respecting triplets of words. From the ultrametric-respecting triplets that we used for analysis, we derived the following interpretation:

1. Derek and John are in a similar relationship relative to Howard.

2. There is a close semantic proximity between family and gun (the latter word, as we have noted above, being used considerably to denote threat, and selfdefence), and then counterposed are Peter, arrow (which appears to be used quite metaphorically by her), football, and the adjective, pudgy. It does appear from this that tensions within the family are associated in her mind with a general, background and ongoing metaphor of a gun.

3. Peter, Mabel and the word horse are all conceived as having distance from Howard and family.

In Murtagh (2014b) further analysis was carried out, along the same lines, on Stephen Fry's Twitter feed in 2009 (when he had about one million followers, whereas he now has 7.4 million). The words we put the spotlight on for such analysis included "party", "young", a few terms related to darts contests, and the locations, Cambridge (England) and LA (Los Angeles). 
In summary, we have proceeded as follows. Firstly, from the given or observed data, we constructed a lantent semantic space, given by our Euclidean-endowed metric factor space produced by Correspondence Analysis. From this factor space, we investigated one aspect of Matte Blanco symmetrization, namely, that the data considered is mapped into an ultrametric, or tree or hierarchy, topology. One of the most straightforward applications of Matte Blanco symmetrization was not pursued here: namely, words or events that are symmetrized. We leave it for future work on appropriate, and preferably vast quantities of data (because of being potentially very interesting), to determine close associations at, or close to, the same chronological time points. Therefore here instead, we addressed the challenging task of finding ultrametric relationships, as being symmetrized in the Matte Blanco sense. As seen, we have established the path to be following in such an endeavour.

\section{Tracking Emotion in Narrative}

In the PhD thesis of Tonti (2012), Matte Blanco's work is taken into the domain of human emotion, which is closely associated with the human subconscious. Emotion is "the component of thought that can be attributed to [...] unconscious rules" (Tonti, 2012, p. 78). "... emotions are a fundamental and building force for cognition, and not just an attribution of affective aspects or a physical response" (p. 164). Emotions are a fundamental constituent of thought and intelligence. They are not juxtaposed to thought and intelligence. Intelligence is not possible without emotion. Emotions are also regulatory functions in our (human) process of segmenting reality (p. 9).

Here we will discuss the tracking of emotion. A full account of this work is in Murtagh and Ganz (2014). We first selected the dialogue interchange between the main protagonists, Rick and Ilsa, in the movie script of Casablanca. There were 150 dialogue expressions, with few exceptions alternating between Rick and Ilsa. Out of the 77 scenes in the movie script, we grouped these dialogue utterances into their scenes, 11 scenes in all. We carried out a Correspondence Analysis on these 11 scenes, crossed by the words they contained. We kept function words, singulars and plurals, due to their potential emotional relevance. We set all upper case to lower case, and removed all punctuation. In the latent semantic space, i.e. the factor space, we looked at the semantic locations - in their full dimensionality in the factor space - for the 11 scenes, and for two selected words betokening emotion, "darling" and "love". Largely we note in Figure 4 the same movement, low values betokening the scene as emotional. Scene 30 is a flashback to Paris and the German occupation, and Scene 70 concerns Ilsa leaving Casablanca with her husband, Laszlo. By appropriate indicator terms, "darling" and "love" being our choice here, our semantic embedding allows us to relate the semantics, both between words used, and the activities in the chronology of the narrative, here the scenes. 


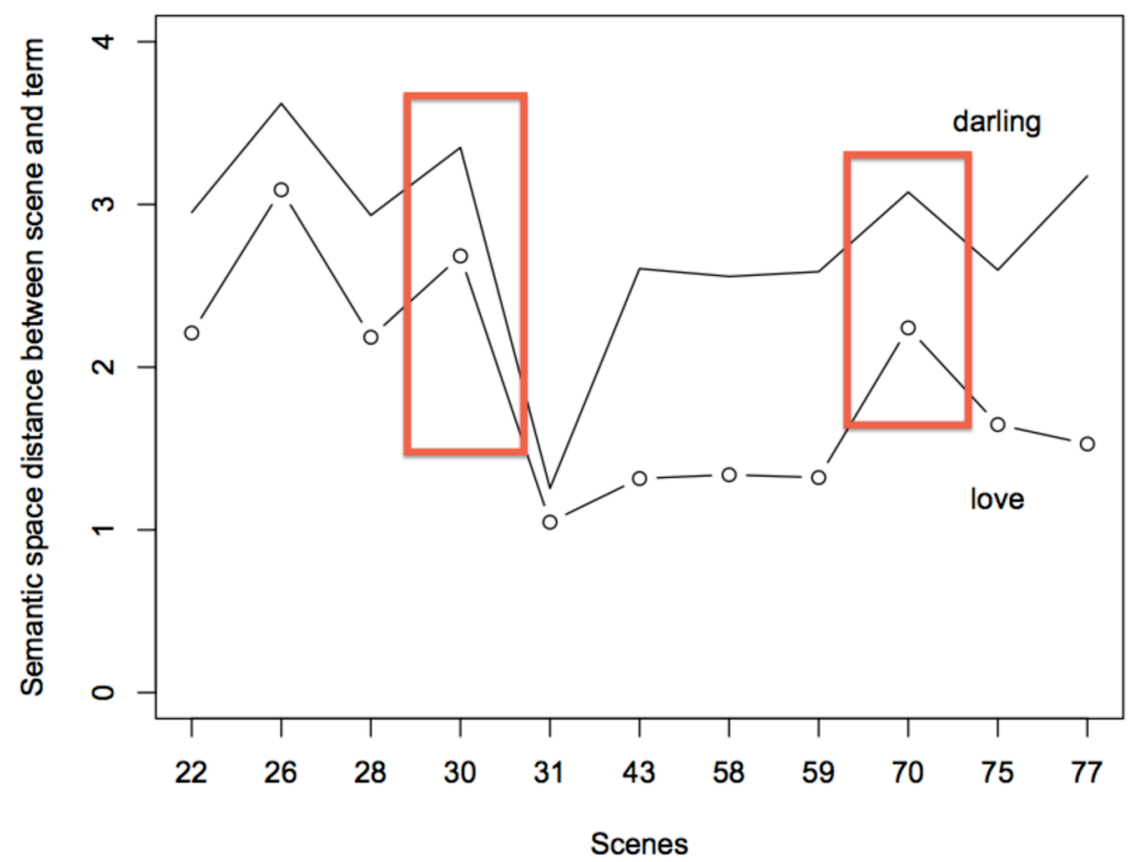

\section{Figure 4}

Casablanca movie: In the full dimensionality factor space, based on all interrelationships of scenes and words, the distance was determined between the word "darling" in this space, with each of the 11 scenes in this space. The same was done for the word "love". The semantic locations of these two words, relative to the semantic locations of scenes 30 and 70 are highlighted with boxes. In these two scenes, there is a pronounced ebbing of emotion.

We next turn attention to the three-way (Emma, her husband Charles, and her lover Rodolphe) relationships in Flaubert's 19th century novel, Madame Bovary. In order to be applied in a very general manner to textual description such as this, we used successive text segments of 20 lines each. Similar data preparation was used as for the Casablanca case study. Sentiment analysis using the words "kiss", "happiness" and "tenderness" was carried out, allowing us to track emotional expression in the chronology of the narrative.

Figure 5 presents an interesting perspective that can be considered relative to the original text. Rodolphe is emotionally scoring over Charles in text segment 1, then again in 3, 4, 5, 6. In text segment 7, Emma is accosted by Captain Binet, giving her qualms of conscience. Charles regains emotional ground with Emma through Emma's father's letter in text segment 10, and Emma's attachment to her daughter, Berthe. Initially the surgery on Hippolyte in text segment 11 draws Emma close to Charles. By text segment 14 Emma is walking out on Charles following the botched surgery. Emma has total disdain for Charles in text segment 15. In text segment 16 Emma is buying gifts for Rodolphe in spite of potentially making Charles indebted. In text segments 17 and 18, Charles' mother is there, with a difficult mother-in-law relationship for Emma. Plans for running away 
ensue, with pangs of conscience for Emma, and in the final text segment there is Rodolphe refusing to himself to leave with Emma.

\section{Emma's evolving state vis-à-vis Rodolphe (lines/circles) and Charles (full lines)}

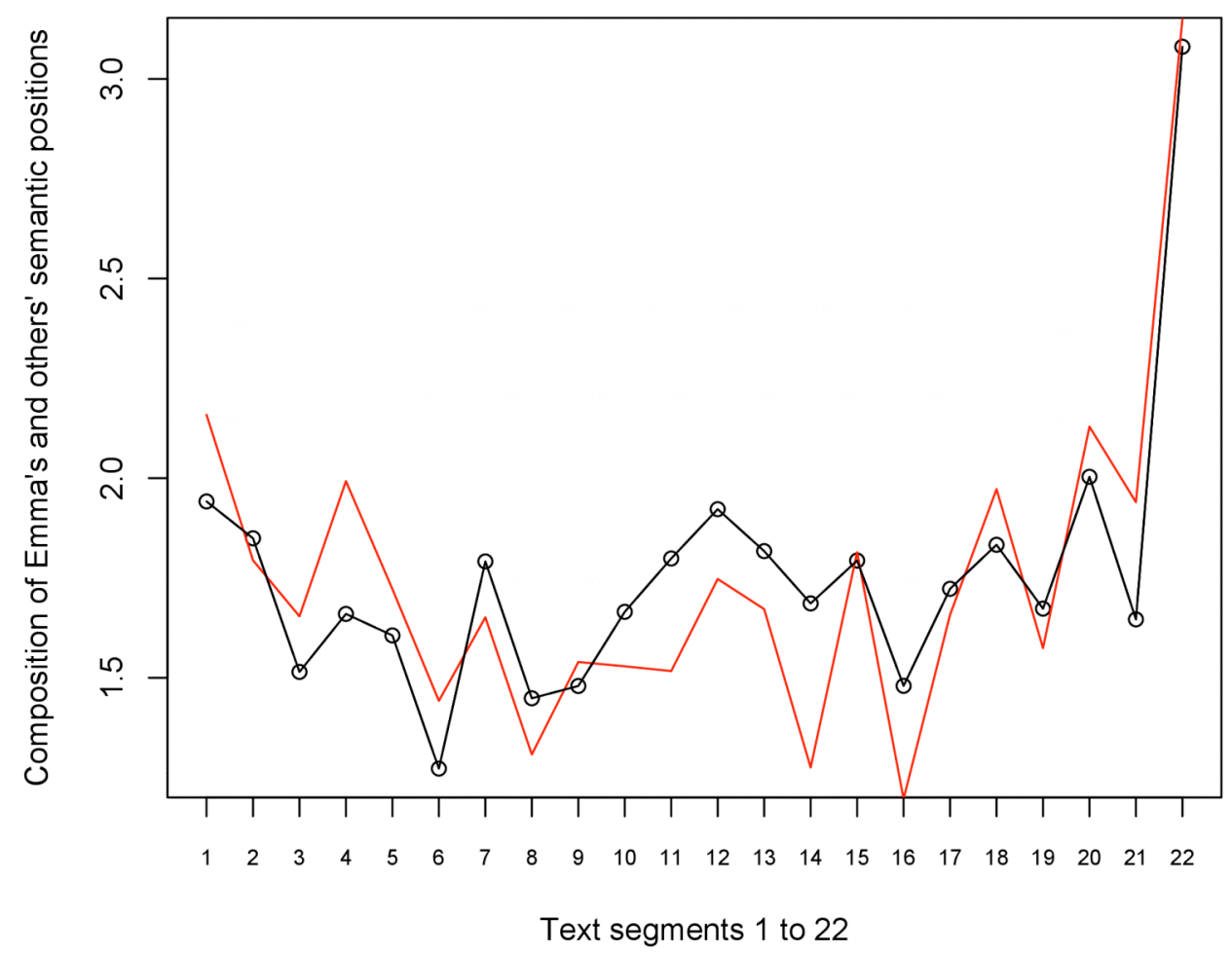

\section{Figure 5}

Madame Bovary novel: The relationship of Emma to Rodolphe (lines/circles, black) and to Charles (full line, red) are mapped out. The text segments encapsulate narrative chronology, that maps approximately into a time axis. Low or small values can be viewed as emotional attachment.

In summary, this data-driven approach allows us to map out the semantic content of our data. It has been shown, too, how we can track out emotional content, and observe its evolution over time. We can also visualize narrative sub-plots, for example using the strength of Emma Bovary's relationship with Charles and with Rodolphe.

\section{Conclusion}

Mathematically modelling Ignacio Matte Blanco's principles of symmetric and asymmetric being in this paper has objectives such as the following: visualization of concepts developed by Matte Blanco, and hence use as an aid for understanding Matte Blanco's work and imparting it to others; and to provide pointers to where and how such mathematical modelling can be instrumentalized, The ambition is even greater, namely 
through Matte Blanco's work to set out, and make extensive use of, the geometry and the topology of mental processes.

It has been shown how the topology of a rooted tree, i.e. an ultrametric topology, can be used as a mathematical model for the structure of the logic that reflects or expresses Matte Blanco's symmetric being, and hence of the reasoning and thought processes involved in unconscious reasoning or in reasoning that is lacking, perhaps entirely, in consciousness or awareness of itself. Such an ultrametric model corresponds to hierarchical clustering that can be induced on empirical data, e.g. text. For Matte Blanco's asymmetric, real-life, and conscious reasoning, a map of empirical data that captures such perceived and defined objects of observation can be formed such that the semantics can be modelled through Euclidean geometry. This furnishes geometrical representation of the latent semantics. Finally we have discussed the passage from metric geometry to ultrametric topology (and vice versa, as is clear from Figure 1). Thus Matte Blanco's bi-logic in its passage from symmetric to asymmetric (in creativity, when emotion comes to lead behaviour, or when trauma dominates behaviour) or vice versa from asymmetric to symmetric (in dream, in repression or in traumatization), in passages both ways between symmetric and asymmetric being, our geometric and topological modelling is enlightening and elucidating. 


\section{References}

Bécue-Bertaut, M., Kostov, B., Morin, A. and Naro, G. (2014), Rhetorical strategy in forensic speeches: multidimensional statistics-based methodology. Journal of Classification, 31, 85-106.

Blasius, J. and Greenacre, M. (Eds.) (2014). Visualization and verbalization of data. Boca Raton, FL: Chapman and Hall/CRC Press.

Bourdieu, P. (1984). Distinction: A social critique of the judgement of taste. Cambridge, MA: Harvard University Press.

Chafe, W. L. (1979). The flow of thought and the flow of language. In T. Givón (Ed.), Syntax and semantics: Discourse and syntax, Volume 12 (pp. 159-181). New York, NY: Academic Press.

Chafe, W. (1994). Discourse, consciousness, and time: The flow and displacement of conscious experience in speaking and writing. Chicago, IL: University of Chicago Press.

Domhoff, G. W. (2002). Using content analysis to study dreams: applications and implications for the humanities. In K. Bulkeley (Ed.), Dreams: A reader on the religious, cultural, and psychological dimensions of dreaming (pp. 307-319). New York, NY: Palgrave.

Domhoff, G. W. (2003). The scientific study of dreams: Neural networks, cognitive development and content analysis. Washington, DC: American Psychological Association.

Domhoff, G. W. (2006). Barb Sanders: Our best case study to date, and one that can be built upon. Retrieved from http://www2.ucsc.edu/dreams/Findings/barb_sanders.html.

DreamBank. Repository of dream reports (2004). Retrieved from http://www.dreambank.net.

Khrennikov, A. (1997). Non-archimedean analysis, quantum paradoxes, dynamical systems and biological models. Dordrecht, The Netherlands: Kluwer.

Khrennikov, A. (2004). Information dynamics in cognitive, psychological, social and anomalous phenomena. Dordrecht, The Netherlands: Kluwer.

Khrennikov, A. (2007). Toward an adequate mathematical model of mental space: Conscious/unconscious dynamics on m-adic trees. Biosystems, 90, 656-675.

Khrennikov, A. (2010). Modelling of psychological behavior on the basis of ultrametric mental space: Encoding of categories by balls. p-Adic Numbers, Ultrametric Analysis, and Applications, 2, 1-20.

Khrennikov, A. (2014, forthcoming). Dynamical processing of information in unconsciousness: ultrametric model.

Lauro-Grotto, R. (2008). The unconscious as an ultrametric set. American Imago, 64, 535-543.

Matte Blanco, I. (1998). The unconscious as infinite sets: An essay in bi-logic. London, UK: Karnac. (Original work published 1975)

McKee, R. (1999). Story - substance, structure, style, and the principles of screenwriting. York, Australia: Methuen.

Murtagh, F. (2005). Correspondence analysis and data coding with Java and R. Boca Raton, FL: Chapman \& Hall/CRC Press.

Murtagh, F., Ganz, A., \& McKie, S. (2009). The structure of narrative: The case of film scripts. Pattern Recognition, 42, 302-312.

Murtagh, F. (2012a). Ultrametric model of mind, I: Review. p-Adic Numbers, Ultrametric Analysis and Applications, 4, 193-206. 
Murtagh, F. (2012b). Ultrametric model of mind, II: Application to text content analysis. p-Adic Numbers, Ultrametric Analysis, and Applications, 4, 207-221.

Murtagh, F. (2014a). Pattern recognition in mental processes: determining vestiges of the subconscious through ultrametric component analysis. In S. Patel, Y. Wang, W.

Kinsner, D. Patel, G. Fariello, \& L. A. Zadeh (Eds.), Proceedings ICCI*CC 2014, 2014 IEEE 13th International Conference on Cognitive Informatics and Cognitive Computing (pp. 155-161). London, IEEE Computer Society Press, Los Alamitos, CA.

Murtagh, F. (2014b, forthcoming). Pattern recognition of subconscious underpinnings of cognition using ultrametric topological mapping of thinking and memory.

International Journal of Cognitive Informatics and Natural Intelligence (IJCINI).

Murtagh, F., \& Ganz. A. (2014, submitted). Pattern recognition in narrative: analysis of narratives of emotion.

Rayner, E. (1995). Unconscious logic: An introduction to Matte Blanco's bi-logic and its uses. Oxford, UK: Routledge.

Schneider, P. (2013). Language usage and social action in the psychoanalytic encounter: discourse analysis of a therapy session fragment. Language and Psychoanalysis, 2, 4-19.

Tonti, M. (2012). Emotions and the unconscious: Modeling and measuring the affective salience of the mind. Unpublished $\mathrm{PhD}$ thesis. Università del Salento. 


\section{Appendix}

\section{Text Analysis as a Proxy for Both Facets of Bi-Logic}

Both conscious or asymmetric reason, and unconscious or symmetric reason, are facets of bi-logic according to Matte Blanco. What he means is that both play a role at different times, that these roles are often complementary, and that the interplay of the two separate domains can be very revealing and instructive. In this Appendix I address the plausibility of appreciable analysis of content of thought processes based on interrelationships that in turn are frequencies of co-occurrence data. Text will be used as a proxy of underlying thinking, reasoning, conscious phenomena and also, every bit as much, representative of the underlying emotional, dreaming, or other unconscious mental processes. What I am seeking is an approach that is deployable and hence usable in practice.

Words are a means or a medium for getting at the substance and energy of a story, notes McKee (1999, p. 179). Ultimately sets of phrases express such underlying issues (the "subtext", as expressed by McKee) as conflict or emotional connotation. Change and evolution are inherent to a plot. Human emotion is based on particular transitions in thought. So this establishes well the possibility that words and phrases are not only taken literally but can appropriately capture and represent such transition. Text, says McKee, is the "sensory surface" of a work of art (counterposing it to the subtext, or underlying emotion or perception). Simple words can express complex underlying reality. Aristotle, for example, used words in common usage to express technically loaded concepts (Murtagh, 2005, p. 169), and Freud did also. Rayner (1995, p. 50) notes the following: "The unconscious largely deals not with particular logically asymmetrically locatable subjects and objects, but with abstract attributes, qualities or notions. Put in another way, these propositional functions are adjectival and adverbial; they lie behind verbal nouns: lovingness, frighteningness and so on." Such words, he notes, are "abstract class attributes, notions or conceptions" and "are the equivalent of the propositional functions of the class".

This has an immediate bearing on the words used in unconscious processes. Rayner (1995, p. 50) notes the "propositional functions or abstract attributes" or "predicate thinking", that underly the unconscious as fundamental constituents. He also briefly exemplifies this through clinical work in schizophrenia and child abuse by adults. One could of course deal with other units of thinking, or reasoning, or unconscious processes, other than through words. Chafe (1979), in relating and establishing mappings between memory and story, or narrative, considered the following units.

1. Memory expressed by a story (memory takes the form of an "island"; it is "highly selective"; it is a "disjointed chunk"; but it is not a book, nor a chapter, nor a continuous record, nor a stream).

2. Episode, expressed by a paragraph.

3. Thought, expressed by a sentence.

4. A focus, expressed by a phrase (often these phrases are linguistic "clauses"). Foci are, "in a sense, the basic units of memory in that they represent the amount of information to which a person can devote his central attention at any one time". 
The "flow of thought and the flow of language" are treated at once, the latter proxying the former, and analyzed in their linear and hierarchical structure as described in other essays in the same volume as Chafe (1979) and in Chafe (1994). In Murtagh (2012a, b) I address the following: Can we attempt to separate out good proxies for symmetrical logic and for asymmetrical logic? To do this, a great number of texts are taken, relating to literature, technical writing, and after-the-fact reporting on unconscious thought processes. 